\title{
Response to "The Use of Extracorporeal Membrane Oxygenation in Clinical Practice" Letter to Editor
}

\author{
Harrison Lang ${ }^{1}$, Mitchell Milanuk ${ }^{1}$, John Brady ${ }^{2}$, Karin Trujillo ${ }^{1}$, Elizabeth Lyden ${ }^{3}$, and \\ HelenMari Merritt Genore ${ }^{1}$ \\ ${ }^{1}$ University of Nebraska Medical Center College of Medicine \\ ${ }^{2}$ University of Pittsburgh Medical Center \\ ${ }^{3}$ University of Nebraska Medical Center
}

May 20, 2020

\begin{abstract}
This is a response to the Letter to Editor received regarding the article "Outcomes of Non-Cardiotomy Patients Requiring Postoperative Extracorporeal Membrane Oxygenation."
\end{abstract}

Dear Dr Harky:

Thank you for your inquiry regarding our manuscript ${ }^{1}$. Our retrospective database captures all ECMO patients from 2006 through Jan 2017. Postoperative ECMO for non-cardiotomy patients has been a recent endeavor and includes 20 perioperative patients supported over a six-year period (2010-2016). These patients were compared with ELSO outcomes and within our own database (practices/selection criteria are most similar within our institution).

We agree it is difficult to make recommendations about decisions for support based on a small cohort of orthotopic liver transplant (OLT) patients, including the referenced case series of eight patients with $38 \%$ survival $(2 / 4 \mathrm{VV} \text { an } 1 / 4 \text { VA survived hospitalization })^{2}$. Cannulation ranged from 0 to 180 days after transplantation. Our manuscript focused only on perioperative support, occurring within index hospitalization, with median time to support 0 days. This may represent a different type of patients, as the initial postoperative period includes unique hemodynamic challenges.

Two other OLT reports include 32 and 18 patients $^{2}$. These larger $V V$-only cohorts report $15 \%$ and $44 \%$ survival. It is unclear how close in proximity of OLT support was initiated. Within The smaller 8 patient case series blends VA and VV support: overall survival was $3 / 8$ patients $(25 \% \mathrm{VA}$ and $50 \% \mathrm{VV})$. In our small experience, perioperative salvage ECMO for OLT has been unsuccessful (0/4).

Our manuscript addresses demographic and preoperative characteristics of the smaller subgroups. We do also discuss the type of ECMO utilized.

OLT: Indications were "cardiac arrest $(\mathrm{n}=2)$, intraoperative cardiogenic shock $(\mathrm{n}=1)$, and postoperative respiratory failure $(\mathrm{n}=1)$. Two patients required VA-ECMO in the setting of ECPR, one patient required VV-ECMO, and one patient fell into the "Other" category as they were supported with RVAD with VV in-line oxygenator."

Thoracic: "Indications were... respiratory failure (n 8) and cardiogenic shock (n 2). Three patients required VA-ECMO, six patients required VV-ECMO, and one patient fell into the "Other" category." We do not have intraoperative details prior to ECMO initiation. 
We agree the use of either VA or VV ECMO respresent distinct underlying disease processes. The PNCECMO and control cohorts were divided into VA and VV ECMO before comparing short and long-term survival rates between the cohorts.

Each case is considered uniquely via multidisciplinary approach with discussion between primary surgeon and anesthesiologist, cardiothoracic surgeon, intensivist, nurses, perfusionists and the patient/family. A teambased approach to difficult situations is essential; we agree this improves outcomes ${ }^{3}$. Likewise, a quality conference occurs at regular intervals to discuss ECMO mortalities and morbidities in an interprofessional setting.

In closing, we agree completely that further studies are needed to define which postoperative patients may benefit. Our manuscript is meant to add to the body of literature available, not to provide guidelines. Thank you for your insightful points and reflections.

\section{References:}

1. Lang H, Milanuk M, Brady J, et al. Outcomes of noncardiotomy patients requiring postoperative extracorporeal membrane oxygenation. J Card Surg. 2020;1-8. https://doi.org/10.1111/jocs.14598

2. Braun H, Pulcrano M, Weber D et al. The Utility of ECMO After Liver Transplantation: Experience at a High-volume Transplant Center and Review of the Literature. Transplantation. 2019 Aug;103(8):15681573.

3. Na SJ, Chung CR, Choi HJ, et al. The effect of multidisciplinary extracorporeal membrane oxygenation team on clinical outcomes in patients with severe acute respiratory failure. Ann Intensive Care. 2018;8(1):31. 2018 Feb 27. doi:10.1186/s13613-018-0375-9 